\title{
Effect of (3- Glycidyloxypropyl)Trimethoxysilane (GOPS) on the Electrical Properties of PEDOT:PSS Films
}

\author{
Anna Håkansson, Shaobo Han, Suhao Wang, Jun Lu, Slawomir Braun, Mats Fahlman, \\ Magnus Berggren, Xavier Crispin and Simone Fabiano
}

\section{Journal Article}

\section{Tweet}

N.B.: When citing this work, cite the original article.

Original Publication:

Anna Håkansson, Shaobo Han, Suhao Wang, Jun Lu, Slawomir Braun, Mats Fahlman, Magnus Berggren, Xavier Crispin and Simone Fabiano, Effect of (3Glycidyloxypropyl)Trimethoxysilane (GOPS) on the Electrical Properties of PEDOT:PSS Films, Journal of Polymer Science Part B, 2017. 55(10), pp.814-820.

http://dx.doi.org/10.1002/polb.24331

Copyright: Wiley: 12 months

http://eu.wiley.com/WileyCDA/

Postprint available at: Linköping University Electronic Press

http://urn.kb.se/resolve?urn=urn:nbn:se:liu:diva-136852

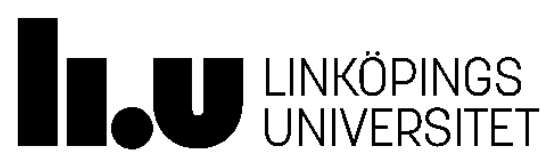




\title{
Effect of (3-glycidyloxypropyl)trimethoxysilane (GOPS) on the electrical properties of PEDOT:PSS films
}

\author{
Anna Håkansson, ${ }^{1}$ Shaobo Han, ${ }^{1}$ Suhao Wang, ${ }^{1}$ Jun Lu, ${ }^{2}$ Slawomir Braun, ${ }^{2}$ Mats Fahlman, ${ }^{2}$ Magnus \\ Berggren, ${ }^{1}$ Xavier Crispin ${ }^{1}$ and Simone Fabiano ${ }^{1}$ \\ ${ }^{1}$ Laboratory of Organic Electronics, Department of Science and Technology, Linköping University, SE-601 74 \\ Norrköping, Sweden \\ ${ }^{2}$ Department of Physics, Chemistry and Biology, Linköping University, SE-581 83, Linköping, Sweden \\ Correspondence to: Simone Fabiano (E-mail: simone.fabiano@liu.se)
}

Additional Supporting Information may be found in the online version of this article.

ABSTRACT: Poly(3,4-ethylenedioxythiophene) doped with poly(styrenesulfonate) (PEDOT:PSS) has been reported as a successful functional material in a broad variety of applications. One of the most important advantages of PEDOT:PSS is its water-solubility, which enables simple and environmental friendly manufacturing processes. Unfortunately, this also implies that pristine PEDOT:PSS films are unsuitable for applications in aqueous environments. To reach stability in polar solvents, (3glycidyloxypropyl)trimethoxysilane (GOPS) is typically used to cross-link PEDOT:PSS. Although this strategy is widely used, its mechanism and effect on PEDOT:PSS performance have not been articulated yet. Here we present a broad study that provides a better understanding of the effect of GOPS on the electrical and electronic properties of PEDOT:PSS. We show that the GOPS reacts with the sulfonic acid group of the excess PSS, causing a change in the PEDOT:PSS film morphology, while the oxidation level of PEDOT remains unaffected. This is at the origin of the observed conductivity changes.

KEYWORDS: PEDOT:PSS, crosslinking, GOPS, film morphology, oxidation level

INTRODUCTION Conducting polymers have attracted enormous interest over the past 20 years, potentially enabling the production of flexible devices that can be processed from solution at low temperatures. The great chemical versatility of conducting polymers allows the modification of their optical, mechanical, energetic and charge transporting properties. Conducting polymers can be dissolved and processed from solution, simplifying the manufacturing process. ${ }^{1,}{ }^{2}$ Furthermore, when water is used as the solvent, the process gets simple and environmental friendly. The water-processable conducting polymer, poly(3,4-ethylenedioxythiophene):polystyrene-sulfonic acid (PEDOT:PSS), was discovered thirty years ago $^{3}$ and is now among the most successful conducting polymers commercially available. PEDOT:PSS is a heavily doped p-type semiconductor, where holes on the positively charged PEDOT chains are compensated by sulfonate anions on the negatively charged PSS chains, acting as the counterions. ${ }^{4}$ It is a mixed ion-electron conductor where cations are transported in the PSS rich domains, and the electrons through the PEDOT. ${ }^{5,6}$ The popularity of this polymer blend arises from many advantageous properties such as high stability in its p-doped form, high conductivity, good film-forming properties, and excellent transparency in the doped state. The electrical conductivity of PEDOT:PSS significantly depends on the morphology, microstructure and the number of counterions that balance the positive doping charges carried by the conjugated polymer chains. ${ }^{4,}$ 7-9 However, due to the high solubility of PSS rich nano-domains in water or other polar solvents, PEDOT:PSS films undergo degradation when exposed to 
such solvents. This exposure is typically encountered for instance when devices are manufactured via multiple layers deposition and in patterning techniques. ${ }^{10,11}$

Yet another very important case where PEDOT:PSS is in contact with polar solvents is the broad area of bioelectronics where PEDOT:PSS, used as the active component in electrochemical devices, has become a key material thanks to its excellent capability to convert ionic signals into electronic signals (and vice versa). ${ }^{2,}{ }^{12}$ Electrochemical devices such as organic electrochemical transistors (OECTs) ${ }^{13,14}$ and organic electronic ion-pump (OEIP) ${ }^{15}$ enable new possibilities of stimulating and recording biological processes and provide for example new solutions for medical healthcare. ${ }^{2}$ In such devices a liquid electrolyte, usually aqueous in bioelectronic applications, is placed in contact with PEDOT:PSS to ensure ionic transport. An usual strategy to obtain water-stable films has been to crosslink the active layer with methoxysilane-based molecules such as (3glycidyloxypropyl)trimethoxysilane (GOPS), which have been proven useful in successfully preventing both dissolution and delamination of PEDOT:PSS films in aqueous solutions. ${ }^{14,} 16,17$ There is yet another emerging research area where the crosslinking of PEDOT:PSS with methoxysilanes is important; stretchable electronics. Indeed, the integration of an elastomeric material, methoxysilane based coupling, into PEDOT:PSS is a promising way to combine both the electrical conductivity and the elasticity required in, for instance, new electronics directly in contact with the skin of the body or even within the body. ${ }^{18}$ Unfortunately, however, addition of GOPS also reduces the electronic as well as the ionic conductivity, ${ }^{16}$ meaning that there is a trade-off between the desirable effects of water-stability or stretch-performance, and the conductivity. Possible reactions between GOPS and PEDOT:PSS have been discussed ${ }^{17}, 19$ but surprisingly, despite the extensive use of methoxysilane-crosslinked PEDOT:PSS, the mechanism of the crosslinking and its effect on the electronic properties of PEDOT is not clearly understood. Thus, to further develop and increase the performance of PEDOT:PSS-based devices it is important to understand the effect of GOPS and its reaction with PEDOT:PSS.

Here we present a detailed investigation of the crosslinking reaction between PEDOT:PSS and GOPS. By combining several characterization methods we studied the property changes of the polymer upon addition of GOPS. We found that the crosslinking does not affect the oxidation level of PEDOT:PSS but instead causes a morphology change through the interaction between GOPS and the excess PSS leading to a reduced charge carrier mobility and hence a reduced conductivity.

\section{EXPERIMENTAL}

\section{Films Fabrication}

PEDOT:PSS (Clevios PH1000 from Heraeus Holding $\mathrm{GmbH}$ ) was filtered through a $0.45 \mu \mathrm{m}$ polyvinylidene fluoride (PVDF) filter and mixed with GOPS at different volume ratio $(0,0.1,0.2$, $0.6,1,5$ and $10 \mathrm{v} / \mathrm{v} \%)$. Also samples of lowconducting PEDOT:PSS (Clevios PVPAI4083 from Heraeus Holding $\mathrm{GmbH}$ ) were prepared following the same procedure. To ensure a uniform distribution of GOPS, the solutions were sonicated for 5 minutes. The PEDOT:PSSGOPS solutions were then spin coated at a speed of $1500 \mathrm{rpm}$ for $30 \mathrm{~s}$, except in the case of films for absorbance measurements where the spin rate was adjusted to obtain similar thickness for all the samples. As different characterization methods were intended to be used, each mixture was coated on several different substrates. For absorbance measurements (370-2500 nm) glass substrates were used. For XPS, UPS and Kelvin probe, indium tin-oxide was used as these measurements required a conductive substrate. For resistance measurements, glass with four $15 \times 1 \mathrm{~mm}$ electrodes with thickness of $6 \mathrm{~nm} \mathrm{Cr}$ and $60 \mathrm{~nm} \mathrm{Au}$, with a $1 \mathrm{~mm}$ distance was used. For TEM measurement, free standing films were 
obtained by first spin coat the polymer on a thin layer of Poly(methyl methacrylate) (PMMA) and then put the samples in acetone where the PMMA dissolved. The films were then supported on copper grids for TEM characterization. To enable the cross-linking process, the spin-coated films were baked in an oven at $140^{\circ} \mathrm{C}$ for 30 minutes. All procedures were conducted in ambient air.

\section{Films Characterization}

Absorbance measurements were conducted using an absorbance spectrometer (PerkinElmer Lambda 900) in the range of 370-2500 nm. Water-stability was evaluated from absorbance measurements at $850 \mathrm{~nm}$ after different incubation periods in water. The resistance was measured by standard four-point-probe measurement using a Keithly 4200-SCS and the film thickness was measured by a Dektak 3ST. The conductivity $(\sigma)$ was calculated from the resistance and the geometry of the film: $\sigma=I(R w t)^{-1}$, where $R$ is the resistance and $I, w$ and $t$ is the length, width and thickness respectively of the film.

Photoemission experiments were carried out using a Scienta ESCA 200 spectrometer in ultrahigh vacuum with a base pressure of $1 \times 10^{-10}$ mbar. A monochromatic Al Ka X-ray

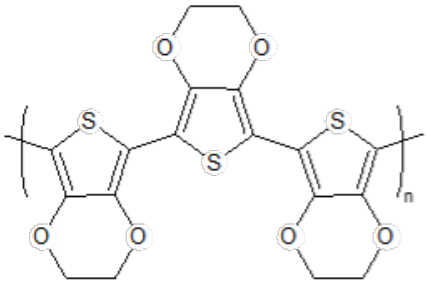

PEDOT

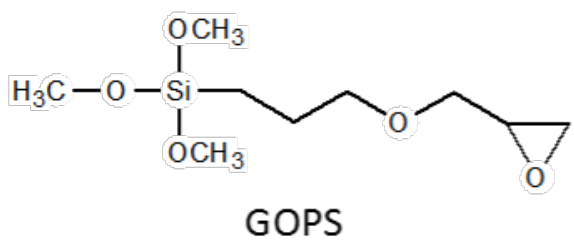

SCHEME 1 Chemical structures of PEDOT:PSS and GOPS source provided photons with energy of 1486.6 $\mathrm{eV}$. The X-ray photoemission spectroscopy (XPS) experimental conditions were set so that the full-width at half-maximum of the clean Au4f7/2 line was $0.65 \mathrm{eV}$. Deconvolution of the $S(2 p)$ spectra was carried out on the basis of a priori knowledge of the chemical composition, and on the results reported in the literature. ${ }^{4}$ During the deconvolution performed on the $S(2 p)$ spectra, the energy difference between the $S\left(2 p_{1 / 2}\right)$ and $S\left(2 p_{3 / 2}\right)$ spin-orbit components was kept at $1.2 \mathrm{eV}$, and the intensity ratio fixed to $1: 2$.

For ultraviolet photoemission spectroscopy (UPS) measurements a standard He-discharge lamp with Hel $21.22 \mathrm{eV}$ was used as excitation source characterized by energy resolutions of 50 meV. Work function was derived from secondary electron cut-off.

Kelvin probe measurements were performed in the high vacuum chamber using a McAllister KP6500 digital Kelvin probe, which features a stainless steel tip (diameter $5 \mathrm{~mm}$ ) as the reference electrode. The resolution of the system was higher than $5 \mathrm{mV}$.

Atomic force microscopy (AFM) measurements were performed in tapping mode using a Veeco Dimension 3100.

TEM characterization was carried out by using a FEl Tecnai G2 20 U-Twin FEG transmission electron microscope operated at $200 \mathrm{kV}$ with a point resolution of $0.19 \mathrm{~nm}$. Bight field images and selected area electron diffraction (SAED) patterns were acquired to obtain microstructural information.

\section{RESULTS}

Scheme 1 shows the chemical structures of PEDOT:PSS and GOPS. When PEDOT:PSS is mixed with GOPS in solution, even at concentration as low as $0.1 \mathrm{v} / \mathrm{v} \%$ GOPS, it forms homogeneous thin films on glass substrates that are insoluble in water. The effect of GOPS 


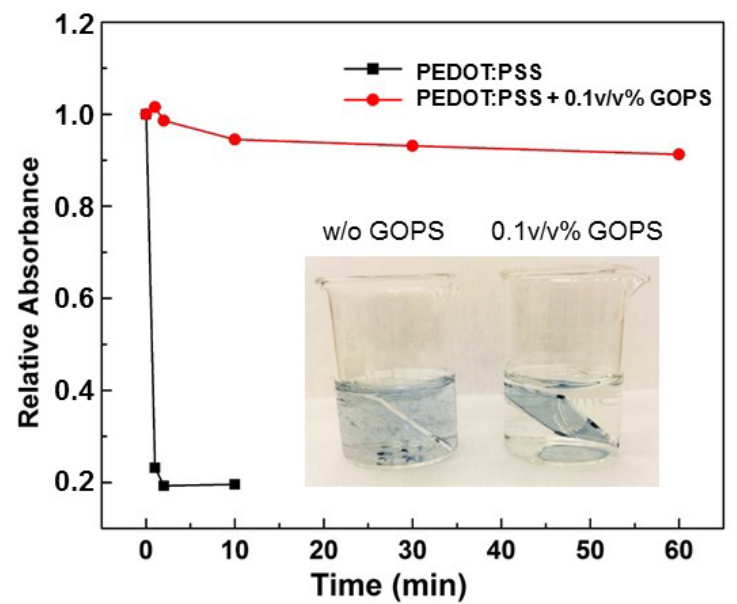

FIGURE 1 Relative absorbance (at $850 \mathrm{~nm}$ ) for pristine PEDOT:PSS and PEDOT:PSS-0.1v/v\% GOPS films for different immersion time in water. The inset shows pristine (left) and GOPStreated (right) PEDOT:PSS films on glass substrates in water.

on the water stability of PEDOT:PSS films is demonstrated by incubating PEDOT:PSS and PEDOT:PSS-0.1v/v\% GOPS thin films in water and measure their absorbance at regular intervals. As the absorbance at $850 \mathrm{~nm}$ only originates from the PEDOT and not from the substrate, its value is proportional to the film thickness. Figure 1 shows a clear difference in thickness evolution during the water incubation as the pristine PEDOT:PSS dissolves immediately while PEDOT:PSS-0.1v/v\% GOPS is hardy affected even after $60 \mathrm{~min}$.

The film morphology of pristine and GOPStreated PEDOT:PSS films was investigated by means of AFM. Figure 2a shows the topography image of untreated PEDOT:PSS film, exhibiting a typical globular grain-like structure with a root mean square roughness of $2.1 \mathrm{~nm}$ (measured over an area of $1 \times 1 \mu \mathrm{m}^{2}$ ). The film roughness decreases with increasing the amount of GOPS (Fig. 2b-d), reaching values as low as $0.83 \mathrm{~nm}$ for PEDOT:PSS-5 v/v\% GOPS (Fig. 2d). In addition, the AFM phase images of pristine and GOPS-treated PEDOT:PSS films reveal a reduced phase separation upon increasing the GOPS content in the blend (Figure S1). A change in the film microstructure is also corroborated by TEM, which reveals a coarsening of the bulk film morphology after GOPS treatment (Figure S2).

Figure 3a shows the effect of GOPS on the electrical conductivity of PEDOT:PSS films spin coated from aqueous dispersion. The conductivity of the pristine PEDOT:PSS is typically of the order of $1 \mathrm{~S} \mathrm{~cm}^{-1}$. These values are well within the performance range reported previously for untreated PEDOT:PSS (PH1000) ${ }^{20}$, assuring the films in this study are representative of the high-performance material. Note also that, although conductivity $>500 \mathrm{~S} \mathrm{~cm}^{-1}$ are obtained when PEDOT:PSS is treated with conductivity enhancement solvents, such as diethylene glycol, ${ }^{21}$ ethylene glycol, ${ }^{20}$ and dimethyl sulfoxide, ${ }^{19}$ we deliberately used here untreated (pristine) PEDOT:PSS to distinguish the effects of solvent treatment from the effect induced by modification of GOPS. Upon addition of only 0.1 v/v\% GOPS, the conductivity significantly drops by one order of magnitude. The electrical conductivity of PEDOT:PSS further decreases with increasing the amount of GOPS in solution and saturates for content larger than $1 \mathrm{v} / \mathrm{v} \%$
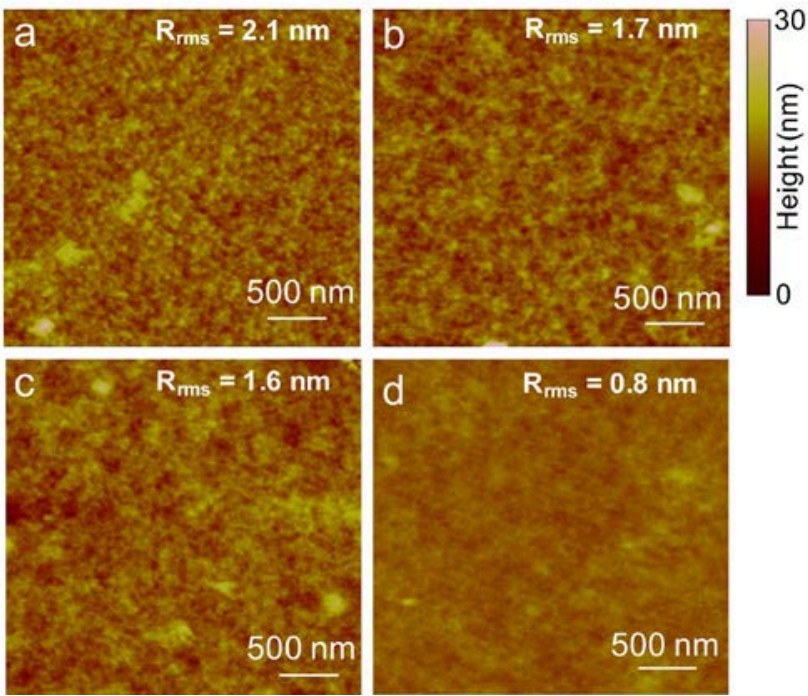

FIGURE 2 AFM images $\left(3 \times 3 \mu \mathrm{m}^{2}\right)$ of pristine (a), $0.2 \mathrm{v} / \mathrm{v} \%$ (b), $1 \mathrm{v} / \mathrm{v} \%$ (c) and 5 v/v\% (d) GOPStreated PEDOT:PSS films, respectively. 

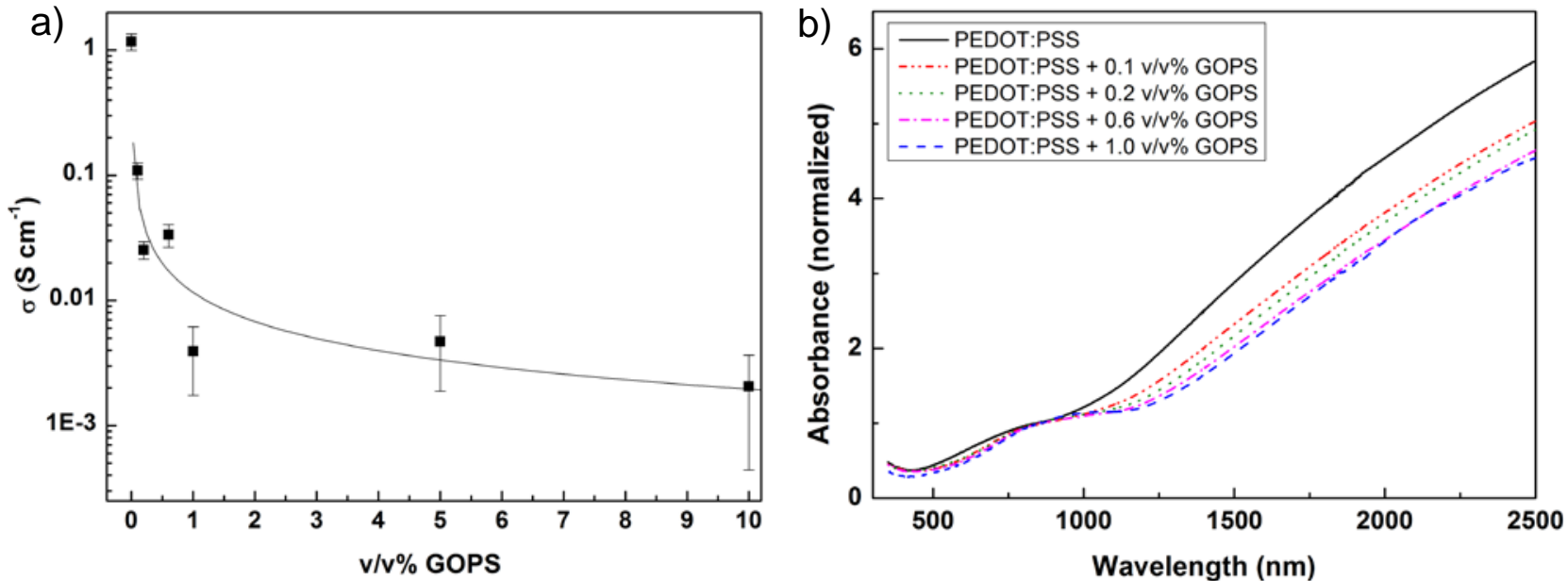

FIGURE 3 a) Electrical conductivity of PEDOT:PSS films as a function of GOPS content ( $/ / v \%)$. Error bars represent standard deviation for at least three samples. b) Absorbance spectrum for PEDOT-PSS with different concentrations of GOPS, normalized for the peak around 850-1050 $\mathrm{nm}$. The decreasing IR absorption upon adding GOPS is attributed to a drop in delocalized charge carrier concentration.

GOPS, reaching values as low as $0.002 \mathrm{~S} \mathrm{~cm}^{-1}$. Note that the GOPS concentration range where most of the electrical conductivity drop occurs is within 0.1 and $1 \mathrm{v} / \mathrm{v} \%$ GOPS, which is the typical value of GOPS content used in OECTs. ${ }^{13}$, 14

The observed decrease in electrical conductivity for GOPS-treated PEDOT:PSS films can be attributed to a reduced charge carrier density in the doped PEDOT or to a decreased charge carrier mobility. The former directly relates to the oxidation level of the PEDOT chains and consequently to their electronic structure. UVvis-NIR absorption measurements provide detailed information about the oxidation state of a conductive polymer as the different type of charge carriers can be identified from their different energy level transitions. ${ }^{22}$ The absorption spectrum of pristine PEDOT:PSS is composed of a weak absorption peak centred at around $850 \mathrm{~nm}$ followed by a broad absorption band that extends far into the near infrared region (Figure $3 \mathrm{~b}$ ). While the peak at lower wavelength is typically associated with localized polarons, the broad IR absorption background is correlated to free delocalized charge carriers and mid-gap states (bipolaron states) of the conducting polymer. To compare the evolution in these two regions upon addition of GOPS, the absorption data shown in Figure $3 b$ is normalized for the peak at around $850 \mathrm{~nm}$. As the GOPS content is increased from 0 to $1 \mathrm{v} / \mathrm{v} \%$, the IR background slightly reduces and the peak, first centred at $850 \mathrm{~nm}$ shifts at lower energy (i.e., about $1050 \mathrm{~nm}$ for 1 v/v\% GOPS). The transition at $1050 \mathrm{~nm}$ is typically associated with uncoupled bipolaron species, suggesting a decreased concentration of delocalized charges to increased disorder. Thus, we can assume the

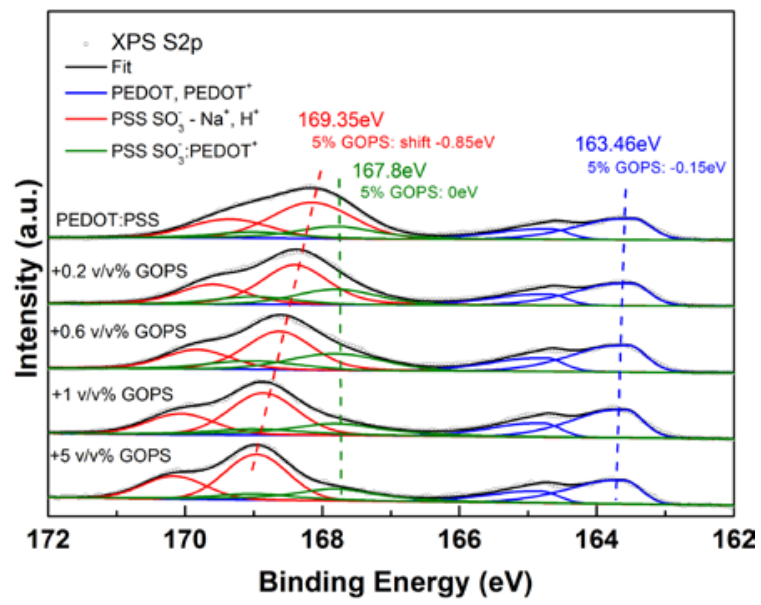

FIGURE 4 Photoelectron spectra of S2p core levels obtained on PEDOT-PSS film with different concentration of GOPS. Values in relation to Fermi energy level. 
charge carrier density in GOPS-treated PEDOT:PSS is unaffected, as a reduction of the doping level would cause a rise of the transition peak at $850 \mathrm{~nm}$ and the appearance of a peak at $600 \mathrm{~nm}$ involving the transformation of polarons into neutral segments. ${ }^{22,23}$

UPS and Kelvin probe measurements corroborate this observation showing no difference in the work function (WF) between pristine and treated PEDOT:PSS (Figure S3 and Table S1 ESI). More specifically, UPS data for pristine PEDOT and PEDOT:PSS- 0.2 v/v\% GOPS (deposited on ITO substrate) reveals WFs of 4.87 and $4.72 \mathrm{eV}$, respectively. For higher concentration of GOPS, the measured WF decreases with scanning time due to the clear influence of charging, as the samples are less conductive thus the obtained data were discarded. Kelvin probe measurements do not face this problem, being a not ionizing method. The WF shift measured by Kelvin probe is significantly smaller than that observed by UPS, with a WF downshift larger than $0.1 \mathrm{eV}$ only for 5 v/v\% GOPS.

To gain insight into the mechanism of the crosslinking reaction between PEDOT:PSS and GOPS, we analyzed the chemical composition of pristine and GOPS-treated PEDOT:PSS films by XPS, focusing in particular on the relative intensities of the characteristic PEDOT and PSS components in S2p core-level line. Figure 4 shows a portion of the XPS spectra corresponding to $S 2 p$ core-level signal of PEDOT:PSS (between 163 and $171 \mathrm{eV}$ binding energy). The $S 2 p$ sulfur atom signal is represented by a doublet due to spin-orbit coupling. The S2p spectra shows two major contributions, one from PEDOT at low binding energy (163-167 eV) and one from PSS at high binding energy (167-171 eV). The sulfur atom in PSS leads to a signal at high binding energy due to the presence of three electronegative oxygen atoms in the sulfonate group, causing a deficit in the electron density of PSS sulfur atom. The PEDOT component of the S2p signal is barely affected by the treatment with GOPS and displays a minor shift by $0.15 \mathrm{eV}$. It is especially worth noting that the tail stretching asymmetrically towards high binding energy, which originates from the presence of positive charges [(bi)polaron defects] delocalized over the doped PEDOT segments, remains unchanged even at GOPS concentration as high as $5 \mathrm{v} / \mathrm{v} \%$. Note that a decrease in the oxidation level, i.e., an increase of neutral PEDOT segments, would lead to a symmetric S2p doublet as typically reported for amine-treated dedoped (neutral) PEDOT; ${ }^{23,}{ }^{24}$ which is not the case here. Those observations support that the PEDOT doping level is not affected by GOPS, and thus a decrease in charge carrier mobility is a likely explanation to the decreased electrical conductivity observed in Figure $3 a$.

More information can be obtained by looking at the PSS contribution to the S2p spectra. The PSS $S 2 p$ signal comprises indeed two components (i.e., two doublets): one originating from the sulfonate $-\mathrm{SO}_{3}{ }^{-}$groups interacting with counter ions (e.g., $\mathrm{Na}^{+}$or $\left.\mathrm{H}^{+}\right)(169.35 \mathrm{eV})$ and one relates to the $-\mathrm{SO}_{3}{ }^{-}$groups interacting with doped PEDOT $(167.8 \mathrm{eV}) .^{25}$ It is clear from the peak deconvolution shown in Figure 4 that the PSS component interacting with the small, free counter ions undergoes a gradual change when increasing the amount of GOPS while the PSS interacting with PEDOT stays intact. This confirms that the doped PEDOT is not affected
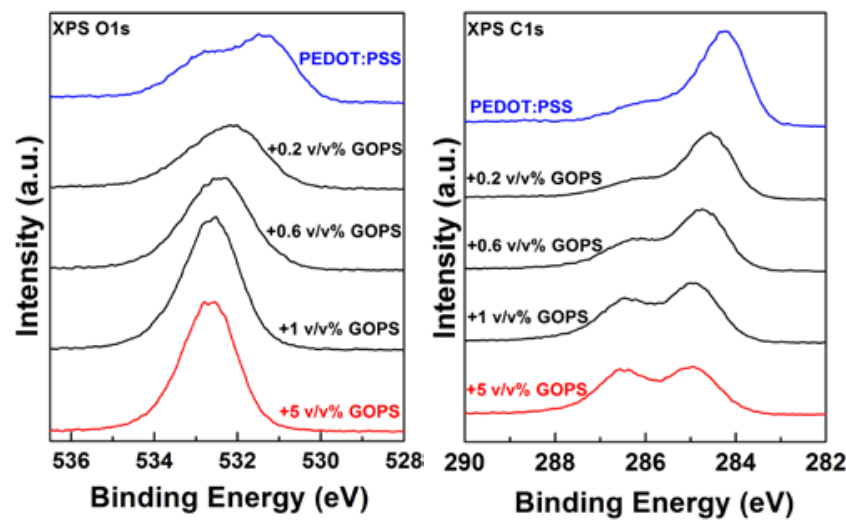

FIGURE 5 Photoelectron spectra of O1s and C1s core levels obtained on PEDOT:PSS film with different concentration of GOPS. 
by GOPS nor is its interaction with $-\mathrm{SO}_{3}{ }^{-}$groups of PSS. The shift of the counter ion-interacting PSS component is very likely due to interaction between the $-\mathrm{SO}_{3}{ }^{-}$groups and GOPS. This could be explained taking into account the very peculiar morphology of PEDOT:PSS films, showing in fact a grain-like structure with conductive PEDOT:PSS-rich cores and an excess of PSS in the outer region. ${ }^{25}$ Hence, the PSS rich domains (being PSS-Na and PSS-H but not PSSPEDOT) would be preferential for the reaction with GOPS.

To further investigate the detail of this interaction between GOPS and PEDOT:PSS, we turn to analyze the changes in the $\mathrm{C} 1 \mathrm{~s}$ and $01 \mathrm{~s}$ core-level signals (Figure 5). The 01s spectra are also strongly affected by addition of GOPS. Based on previous studies, ${ }^{25}$ the low binding energy contribution (531.4 eV) in pristine PEDOT:PSS sample is assigned to the sulfonate groups in PSS-Na and PSS-H while the high binding energy peak at $532.6 \mathrm{eV}$ is attributed to oxygen atoms in the ring formed with the ethylendioxy segment in PEDOT. Upon addition of GOPS, the sulfonate PSS contribution gradually shifts by $1.4 \mathrm{eV}$ towards high binding energies merging into a large signal at $532.8 \mathrm{eV}$. Interestingly, the C1s spectra reveals a similar effect for the carbon atoms on PSS $(284.2 \mathrm{eV}$ peak) displaying a shift of $0.7 \mathrm{eV}$ towards high binding energy by addition of GOPS. Of course, the addition of the GOPS, containing carbon atoms bonded to oxygen atoms is visible with the new contribution at $286.6 \mathrm{eV}$, increasing with the GOPS content. To rationalize the significant shift to high binding energy for the $\mathrm{C} 1 \mathrm{~s}, \mathrm{O} 1 \mathrm{~s}$ and S2p signals of PSS, we need to first remind that the negative charge of the anionic group is actually delocalized on all the atoms on the sulfonate and benzene ring in PSS. Hence, the reaction with GOPS uses this delocalized electron to create a new bond between the sulfonate and the GOPS (see Figure 6). As a result, the electron density on all the atoms of the sulfonate and benzene ring decreases significantly and this contributes to a shift of the $\mathrm{C} 1 \mathrm{~s}, \mathrm{O} 1 \mathrm{~s}$ and $\mathrm{S} 2 \mathrm{p}$ towards higher binding energy.

\section{DISCUSSION}

Based on the XPS results and previous described grain-structure model of PEDOT:PSS, we propose a general structure of the system and a mechanism for the cross-linking reaction of PEDOT:PSS with GOPS, as reported in Figure 6. Methoxysilane groups, such as those contained in the GOPS molecule, are known to be very reactive when in contact with water, or moisture. ${ }^{26},{ }^{27}$ In this condition the methoxysilane group undergoes condensation by losing the protective group directly connected to the Si atom (that is, $-\mathrm{OCH}_{3}$ in the case of GOPS) and form silanols, $\mathrm{SiOH}$. The latter can then, in excess of water, easily reacts further with other alcohols or hydroxyl groups through water condensation thus creating stable siloxane bonds. ${ }^{26}$ This suggests that the methoxysilane groups of GOPS may bind to each other and/or to the glass substrate, building a 3D network. The epoxy ring, positioned at other end of the GOPS molecule, is known to be reactive as well and reacts easily with amides, alcohols, thiols and acids. ${ }^{28}$ XPS results shows that only excess PSS (that is, PSS which is not interacting with PEDOT) is affected upon addition of GOPS. Each PSS-unit contains a benzenesulfonic acid that can react with the epoxy ring of GOPS.

Thus, one of the possible cross-linking reaction mechanism between PEDOT:PSS and GOPS (Figure 6) occurs between the $-\mathrm{SO}_{3}$ groups of the excess PSS and the epoxy ring of GOPS, whereas the GOPS methoxysilane group more likely bonds either to the methoxysilane group of another GOPS molecule, and/or to the glass substrate. This would explain why GOPS prevents both dissolution of the PEDOT:PSS film and delamination from the substrate. 

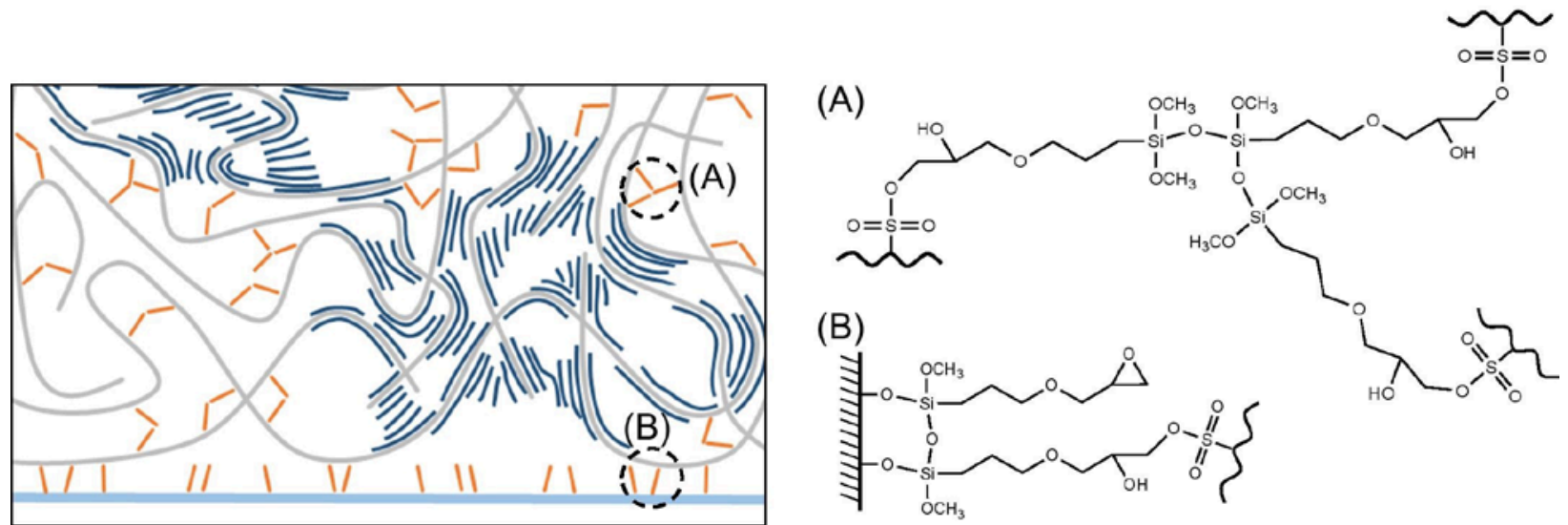

FIGURE 6 Schematic illustration of the system of GOPS-crosslinked PEDOT:PSS. GOPS is only reacting with the parts of the PSS-chains (grey) not interacting with PEDOT (dark blue). The zoom in shows three main interactions involving GOPS; GOPS-PSS, GOPS-GOPS and GOPS-glass. (A) shows the chemical bonds between $-\mathrm{SO}_{3} \mathrm{H}$ group of PSS and the epoxy ring of GOPS, as well as the chemical bond between three GOPS molecules. (B) shows the chemical bond between hydroxyl groups at the glass surface and the methoxysilane groups of GOPS.

It is worth mentioning here that GOPS-treated PEDOT:PSS films typically show a reduced ionic conductivity, compared to the pristine PEDOT:PSS films. ${ }^{16}$ This drop in ionic conductivity is larger than the observed decrease in electrical conductivity, corroborating the fact that GOPS reacts primarily with PSS and is located in the PSS-rich regions, where ions move. The PEDOT-rich conducting grains are thus electrically insulated from each other upon addition of GOPS since the latter insulating molecules are located mostly in the PSS-rich regions. As a result, the electrical conductivity suddenly drops by adding the GOPS and reaches low values. Indeed, in the range 0 to $1 \mathrm{v} / \mathrm{v} \%$ GOPS, the PSS units outnumber GOPS molecules while they are equal in number at around $1 \mathrm{v} / \mathrm{v} \%$, where the conductivity starts to saturate. At the concentration of $5 \mathrm{v} / \mathrm{v} \%$ GOPS, the number of GOPS molecules have by far exceeded the number of PSS units, suggesting a lack of binding sites for GOPS as the origin of the electrical conductivity saturation. This hypothesis can be further supported by studying the conductivity decay of a PEDOT:PSS having a higher content of PSS (PVPAI4083, 1:6 PEDOT:PSS ratio), and subjected to the same GOPS treatment (Figure S4). In that case, the PSS units outnumber GOPS molecules even at 1 v/v\% GOPS and a saturation is only reached at larger GOPS concentrations.

\section{CONCLUSIONS}

The conducting polymer PEDOT:PSS can be made water-stable and elastic by using a methoxysilane-crosslinking agent. However, the crosslinking strategy leads to a reduction of the ionic and electrical conductivity. The interplay between those properties and the mechanism beyond those properties have been investigated. We followed the evolution of the electrical conductivity, absorbance spectrum, work function and morphology of PEDOT:PSS films as a function of the content of methoxysilane GOPS. The electrical conductivity of PEDOT:PSS decreased with increasing the amount of GOPS to a certain level where it saturated. We propose that this decline is due 
to a change in the morphology as a result of the reaction between GOPS and PSS matrix surrounding the highly conducting PEDOT:PSS grains. This is supported by XPS and absorption spectra indicating that the oxidation level of PEDOT as well as the PEDOT:PSS interaction are unaffected by GOPS. On the contrary, the GOPS is located in the PSS rich domain and chemically reacts with the sulfonate groups, causing a drop of the PEDOT:PSS electrical conductivity. This also provides an explanation to the reduced ionic conductivity previously observed in PEDOT:PSS upon addition of GOPS. ${ }^{16}$ This study will help researchers have a better understanding of the effect of GOPS on PEDOT:PSS properties and develop efficient water-stable conducting polymers useful for applications in the fields of bioelectronics, electrochemical devices and stretchable electronics.

\section{ACKNOWLEDGEMENTS}

The authors acknowledge the Swedish Foundation for Strategic Research (Synergy project), the Swedish Energy Agency, China Scholarship Council and the Advanced Functional Material program at Linköping University. The authors also thank Dr. Ujwala Ail for help with TEM samples preparation.

\section{REFERENCES}

1. A. J. Heeger, Synthetic Metals, 2002, 125, 23-42.

2. J. Rivnay, R. M. Owens and G. G. Malliaras, Chemistry of Materials, 2014, 26, 679-685.

3. L. J. Groenendaal, Friedrich; Freitag, Dieter; and H. R. Pielartzik, John R. Advanced Materials, 2000, 12, 481-494.

4. X. Crispin, S. Marciniak, W. Osikowicz, G. Zotti, A. W. D. van der Gon, F. Louwet, M. Fahlman, L. Groenendaal, F. De Schryver and W. R. Salaneck, Journal of Polymer Science Part B: Polymer Physics, 2003, 41, 2561-2583.

5. H. Wang, U. Ail, R. Gabrielsson, M. Berggren and X. Crispin, Advanced Energy Materials, 2015, 5, n/a-n/a.

6. L. Ouyang, C. Musumeci, M. J. Jafari, T. Ederth and O. Inganäs, ACS Applied Materials \& Interfaces, 2015, 7, 1976419773

7. S. K. M. Jönsson, J. Birgerson, X. Crispin, G. Greczynski, W. Osikowicz, A. W. Denier van der Gon, W. R. Salaneck and M. Fahlman, Synthetic Metals, 2003, 139, 1-10.

8. A. N. Aleshin, S. R. Williams and A. J. Heeger, Synthetic Metals, 1997, 94, 173-177.

9. X. Hou, Q. Li, T. Cheng, L. Yu, F. Wang, J. Lin, S. Dai, Y. Li and Z. a. Tan, J. Mater. Chem. A, 2015, 3, 18727-18734.

10. S. Zhang, E. Hubis, C. Girard, P. Kumar, J. DeFranco and F. Cicoira, J. Mater. Chem. C, 2016, 4, 1382-1385.

11. S. Ouyang, Y. Xie, D. Zhu, X. Xu, D. Wang, T. Tan and H. H. Fong, Organic Electronics, 2014, 15, 1822-1827.

12. M. Berggren and A. Richter-Dahlfors, Advanced Materials, 2007, 19, 32013213.

13. K. Tybrandt, S. B. Kollipara and M. Berggren, Sensors and Actuators $B$ : Chemical, 2014, 195, 651-656.

14 L. Kergoat, B. Piro, D. T. Simon, M. C. Pham, V. Noel and M. Berggren, Adv Mater, 2014, 26, 5658-5664.

15. J. Isaksson, P. Kjall, D. Nilsson, N. D. Robinson, M. Berggren and A. RichterDahlfors, Nat Mater, 2007, 6, 673-679.

16. E. Stavrinidou, P. Leleux, H. Rajaona, D. Khodagholy, J. Rivnay, M. Lindau, S. Sanaur and G. G. Malliaras, Adv Mater, 2013, 25, 4488-4493.

17. O. Berezhetska, B. Liberelle, G. De Crescenzo and F. Cicoira, J. Mater. Chem. B, 2015, 3, 5087-5094.

18. Z. U. Khan, J. Edberg, M. M. Hamedi, R. Gabrielsson, H. Granberg, L. Wagberg, I. 
Engquist, M. Berggren and X. Crispin, Adv Mater, 2016, 28, 4556-4562.

19. S. Zhang, P. Kumar, A. S. Nouas, L. Fontaine, $\mathrm{H}$. Tang and F. Cicoira, APL Materials, 2015, 3, 014911.

20. Z. Li, F. Qin, T. Liu, R. Ge, W. Meng, J. Tong, S. Xiong and Y. Zhou, Organic Electronics, 2015, 21, 144-148.

21. X. Crispin, F. L. E. Jakobsson, A. Crispin, P. C. M. Grim, P. Andersson, A. Volodin, C. van Haesendonck, M. van der Auweraer, W. R. Salaneck and M. Berggren, Chemical Materials, 2006, 18, 4354-4360.

22. O. Bubnova and X. Crispin, Energy \& Environmental Science, 2012, 5, 9345.

23. S. Fabiano, S. Braun, X. Liu, E. Weverberghs, P. Gerbaux, M. Fahlman, M. Berggren and X. Crispin, Adv Mater, 2014, 26, 6000-6006.
24. O. Bubnova, Z. U. Khan, A. Malti, S. Braun, M. Fahlman, M. Berggren and X. Crispin, Nat Mater, 2011, 10, 429-433.

25. G. Greczynski, T. Kugler, M. Keil, W. Osikowicz, M. Fahlman and W. R. Salaneck, Journal of Electron Spectroscopy and Related Phenomena, 2001, 121, 1-17.

26. U. S. Maskos, Edwin M., Nucleic Acids Research, 1992, 20, 1679-1684.

27. F. de Buyl, in Inorganic Polymers, ed. R. G. de Jaeger, Mario, Nova Science Publishers, New York, 2007, ch. Silicones in Industrial Applications.

28. J. B. Piehler, Andreas; Valiokas, Ramunas; Liedberg, Bo; Gauglitz, Günter, Biosensors and Bioelectronics, 2000, 15, 473-481. 


\section{GRAPHICAL ABSTRACT}

Anna Håkansson, Shaobo Han, Suhao Wang, Jun Lu, Slawomir Braun, Mats Fahlman, Magnus Berggren, Xavier Crispin and Simone Fabiano

Effect of (3-glycidyloxypropyl)trimethoxysilane (GOPS) on the electrical properties of PEDOT:PSS films

The conducting polymer PEDOT:PSS is an useful and well-studied functional material. This mixed ionelectron conductor has gain lots of popularity from its water-solubility, enables simple processing. This feature unfortunately implies that solid structures dissolves when operating in aqueous environments. To avoid this, methoxysilane-based crosslinkers are often added, making the polymer films water-stable, but also reducing the conductivity significantly. This study helps to understand this crosslinking mechanism and shows how it affect the properties of PEDOT:PSS.

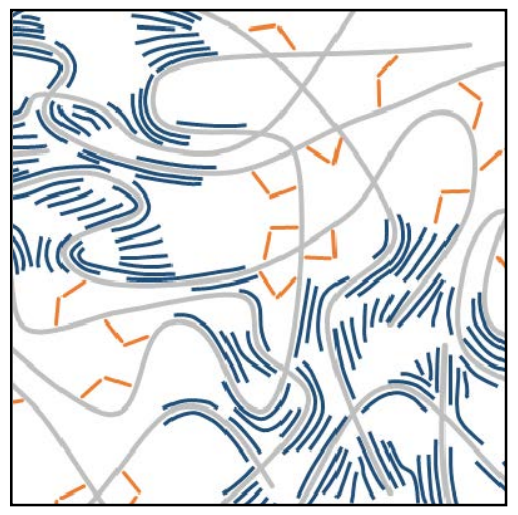


Supporting Information

\section{Effect of (3-glycidyloxypropyl)trimethoxysilane (GOPS) on the electrical properties of PEDOT:PSS films}

Anna Håkansson, ${ }^{1}$ Shaobo Han, ${ }^{1}$ Suhao Wang, ${ }^{1}$ Jun Lu, ${ }^{2}$ Slawomir Braun, ${ }^{2}$ Mats Fahlman, ${ }^{2}$ Magnus Berggren, ${ }^{1}$ Xavier Crispin ${ }^{1}$ and Simone Fabiano ${ }^{1}$

${ }^{1}$ Laboratory of Organic Electronics, Department of Science and Technology, Linköping University, SE-601 74 Norrköping, Sweden

${ }^{2}$ Department of Physics, Chemistry and Biology, Linköping University, SE-581 83, Linköping, Sweden

Correspondence to: Simone Fabiano (E-mail: simone.fabiano@liu.se) 

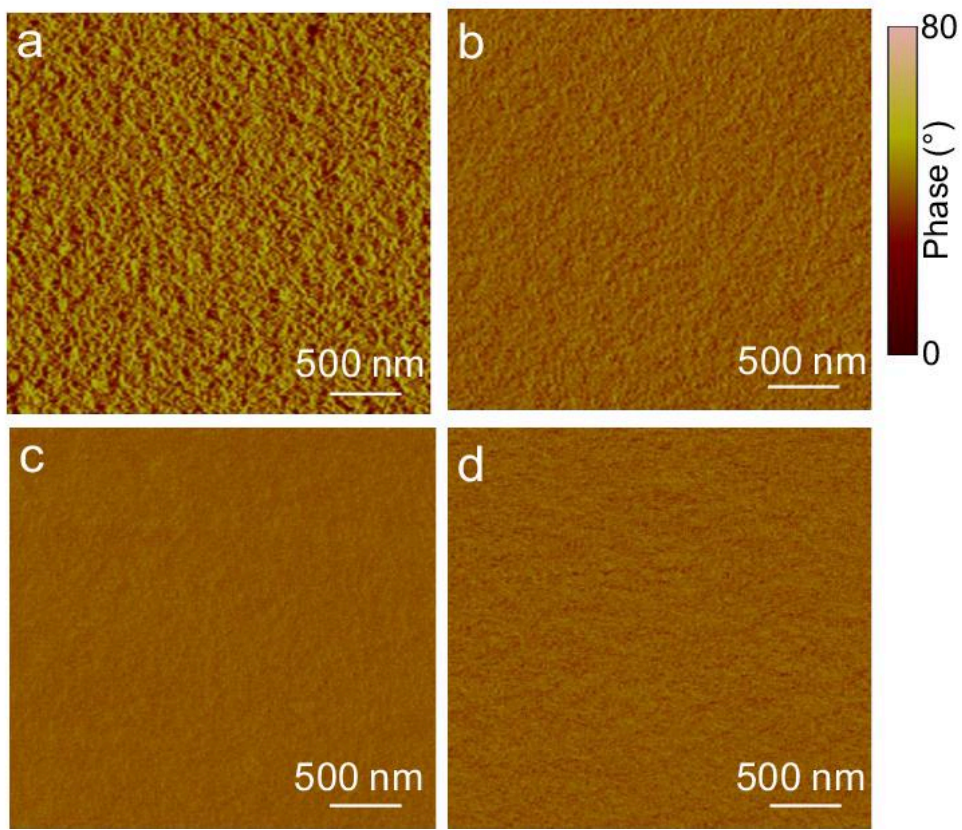

Figure S1: AFM phase images $\left(3 \times 3 \mu \mathrm{m}^{2}\right.$ ) of pristine (a), $0.2 \mathrm{v} / \mathrm{v} \%$ (b), $1 \mathrm{v} / \mathrm{v} \%$ (c) and $5 \mathrm{v} / \mathrm{v} \%$ (d) GOPStreated PEDOT:PSS films, respectively.

Pristine PEDOT:PSS

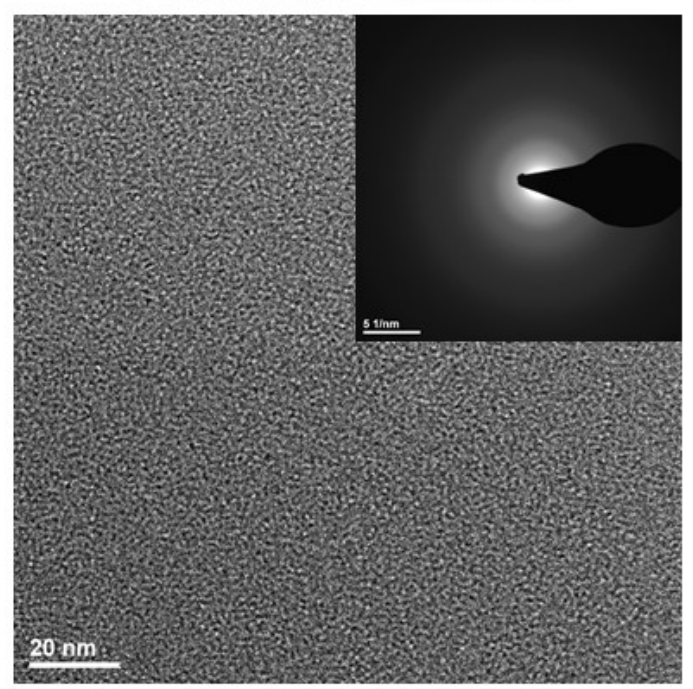

PEDOT:PSS + 1 v/v\% GOPS

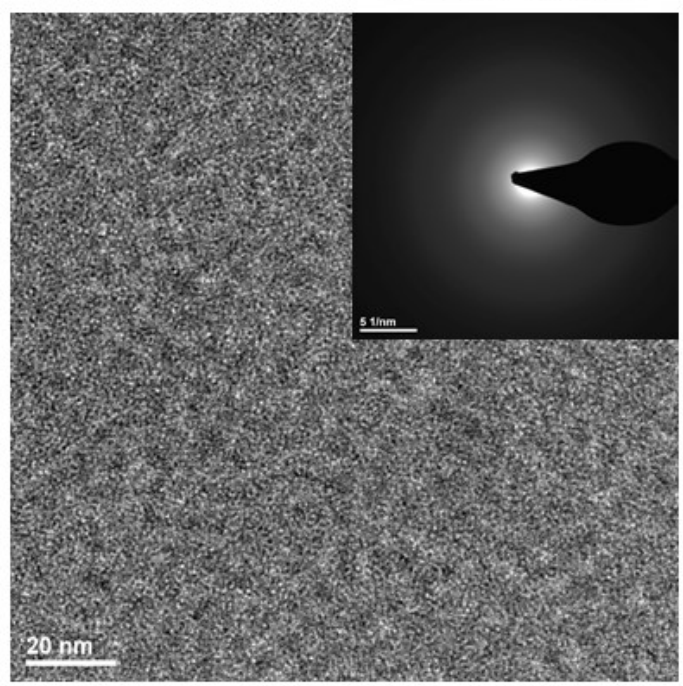

Figure S2: Results from TEM analysis of pristine PEDOT:PSS and PEDOT:PSS $+1 \mathrm{v} / \mathrm{v} \%$ GOPS. There is a clear difference in the morphology where the GOPS treated sample shows a larger grain structure compared to the pristine. The insets shows selected area electron diffraction patterns (SAED) where the circular pattern means the samples are amorphous. 
Table S1: Work function of PEDOT:PSS thin film samples of various GOPS concentrations, investigated by UPS and Kelvin Probe. The higher GOPS concentration samples for UPS were influenced by charging and were discarded.

\begin{tabular}{|l|l|l|}
\hline sample & $\begin{array}{l}\text { UPS (ITO substrate), } \\
\text { relative shift (eV) }\end{array}$ & $\begin{array}{l}\text { Kelvin Probe (glass substrate), } \\
\text { relative shift (eV) }\end{array}$ \\
\hline pristine & reference sample & reference sample \\
\hline $0.2 \%$ GOPS & -0.15 & -0.023 \\
\hline $0.6 \%$ GOPS & $-0.15:-0.25$ & -0.097 \\
\hline $1 \%$ GOPS & $-0.39:-0.5$ & -0.071 \\
\hline $5 \%$ GOPS & $-0.4:-0.49$ & -0.236 \\
\hline
\end{tabular}

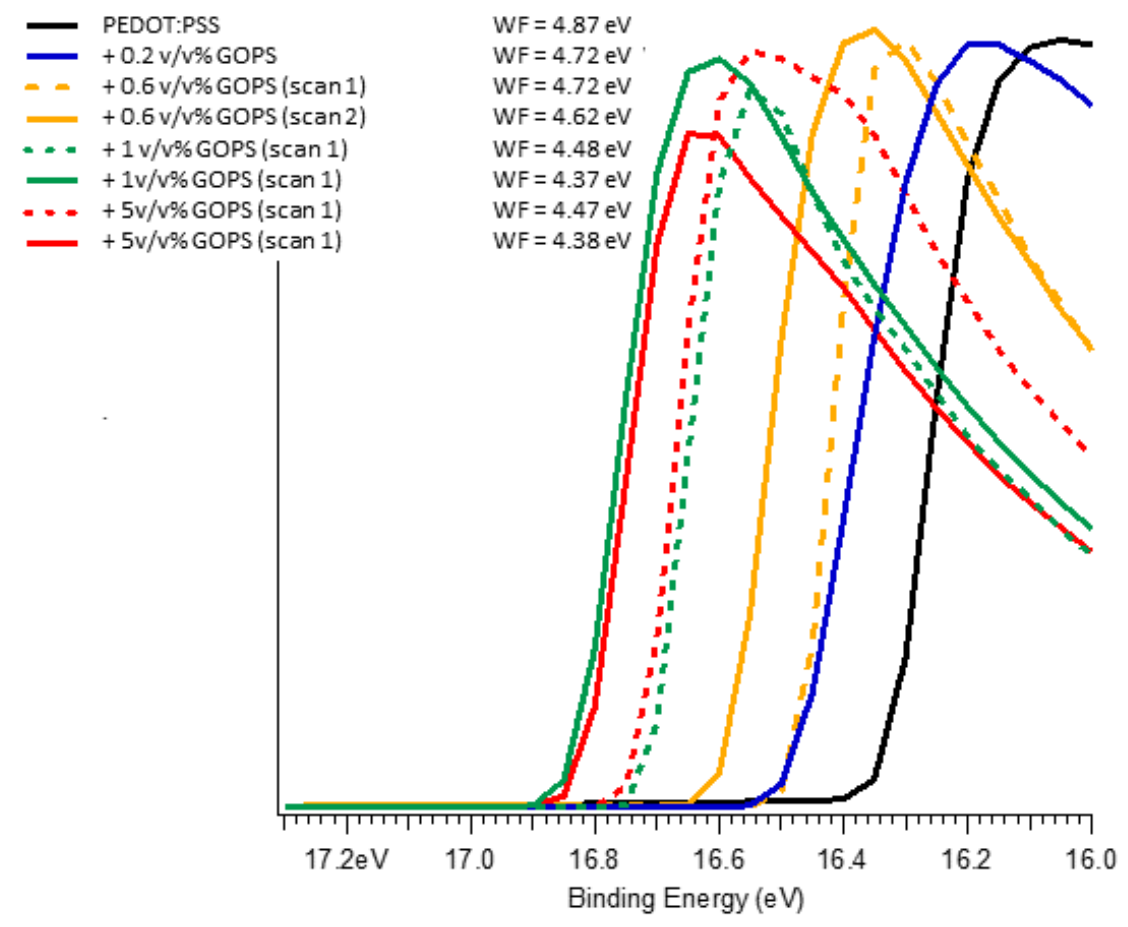

Figure S3: UPS data for pristine PEDOT and PEDOT- 0.2\% GOPS (on ITO) reveals work function of 4.87 and $4.72 \mathrm{eV}$, respectively. For the higher concentration of GOPS there is unfortunately clear influence of charging (as the samples are less conductive): the work function measured decreases with scanning time. 


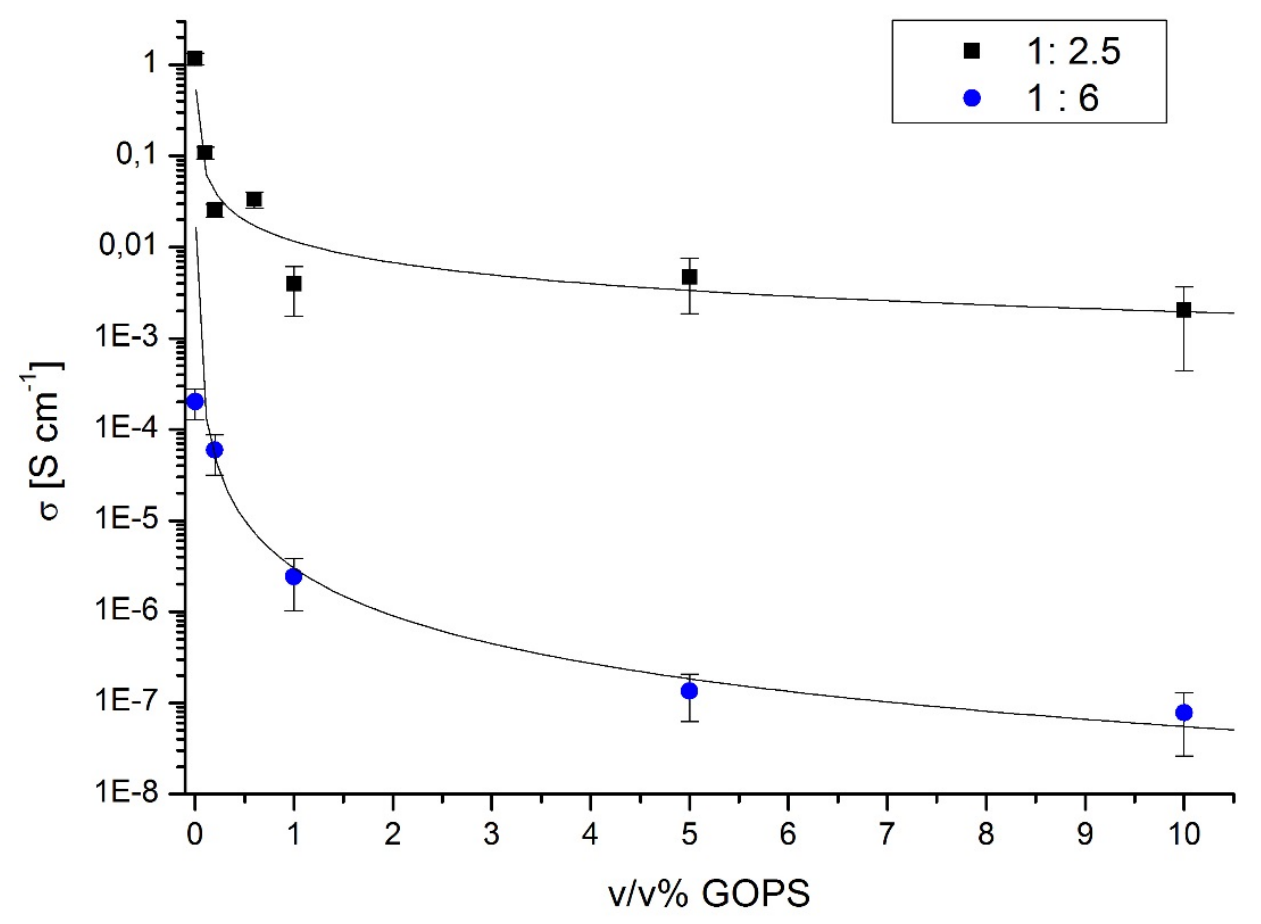

Figure S4: The electrical conductivity decay for different PEDOT:PSS-ratio upon addition of GOPS. While the conductivity of the polymer of 1:2.5 PEDOT:PSS ratio saturates around $1 \mathrm{v} / \mathrm{v} \%$ GOPS, it continue to decrease in case of a high PSS content polymer with a 1:6 PEDOT:PSS ratio even at higher GOPScontents. 